\title{
Clinical Characteristics of Labyrinthine Concussion
}

\author{
Mi Suk Choi, See-Ok Shin, Je Yeob Yeon, Young Seok Choi, Jisung Kim and Soo Kyoung Park \\ Department of Otorhinolaryngology-Head and Neck Surgery, College of Medicine, Chungbuk National University, Cheongju, Korea
}

Received November 30, 2012

Revised February 15, 2013

Accepted February 25, 2013

\begin{abstract}
Background and Objectives: Inner ear symptoms like hearing loss, dizziness or tinnitus are often developed after head trauma, even in cases without inner ear destruction. This is also known as labyrinthine concussion. The purpose of this study is to determine the clinical manifestations, characteristics of audiometry and prognostic factors of these patients. Materials and Methods: We reviewed the medical records of the 40 patients that had been diagnosed as labyrinthine concussion from 1996 to 2007. We studied the hearing levels in each frequency and classified them according to type and degree of hearing loss. Rates of hearing improvement were evaluated according to age, sex, hearing loss type, degree and presence of dizziness or tinnitus. To find out any correlation between hearing improvement and these factors, we used $\chi^{2}$ test or Fisher's exact test. Results: Bilateral hearing loss was observed in 22 patients, and unilateral hearing loss in 18 patients. There were 4 (6.5\%) ascending, 34 (54.8\%) descending, 24 (38.7\%) flat type hearing loss, which indicated hearing loss was greater in high frequencies than low frequencies. Among 62 affected ears, 20 (32.3\%) gained improvement, and it was achieved mainly in low frequencies. There were only 2 ears with dizziness in 20 improved ears and among 20 dizziness accompanied ears, also only 2 ears were improved. Conclusions: High frequencies are more vulnerable to trauma than low frequencies. The hearing gain is obtained mainly in low frequencies, and association with dizziness serves poor prognosis.
\end{abstract}

Korean J Audiol 2013;17:13-17

KEY WORDS: Labyrinthine concussion · Post-concussion syndrome · Hearing loss Sensorineural · Post-traumatic.

\section{Introduction}

Head trauma sometimes causes inner ear damages presenting symptoms like hearing loss, tinnitus and dizziness. ${ }^{1,2)} \mathrm{Di}$ rect injuries to the inner ear, fracturing along the cochlea and vestibule, as a matter of course, bring inner ear symptoms. However, also there are some patients who complaint about these symptoms without a sign of direct injury to the labyrinth on radiologic studies. This such clinical entity is described as labyrinthine concussion previously, and it is defined as high frequency sensorineural hearing loss with or without vestibular symptoms following head trauma without a definite labyrinthine fracture. ${ }^{3)}$ This type of hearing loss also termed inner ear concussion, commotion labyrinthitis, and otitis interna vasomotoria. ${ }^{4)}$ Although various studies have tried to explain the hearing loss that appears after head trauma, the mechanism of injury is not clear. ${ }^{5-8)}$

Although otolaryngologists often face labyrinthine concus- sion patients, very limited information about these patients have been reported in medical literatures. This study is designed to discuss about clinical manifestations, characteristics of audiometry and prognostic factors of labyrinthine concussion patients.

\section{Materials and Methods}

We reviewed the medical records of the patients who had been diagnosed in Chungbuk National University Hospital as hearing loss developed after head trauma from August, 1996 to June, 2007. Every patient underwent thorough physical exam, audiologic exam including pure tone audiometry (PTA) and speech audiometry. Also, temporal bone and brain computed tomography were proceeded to evaluate accompanied skull fracture, intracranial bleeding or inner and middle ear injury. Patients with central nervous system symptom such as disorientation, dysarthria, ataxia were excluded. Among them, 
40 (62 ears) patients who had sensorineural type hearing loss on PTA without any abnormal findings on physical and radiologic exams, were enrolled in this study. The inclusion criteria for labyrinthine concussion were as follows: the patients who had 1) previous history of head trauma without any abnormal finding on radiologic studies, 2) inner ear symptoms such as hearing disturbance with or without dizziness and tinnitus after trauma, 3) no history of hearing disturbance before the trauma, 4) sensorineural hearing loss on PTA greater than $25 \mathrm{~dB}$ according to AAO-HNS standard. Any patients who had history of previous hearing loss or chronic otitis media, and sign of conductive hearing loss or perilymphatic fistula were excluded. All patients were followed up for at least 12 months with PTA on a regular basis, without any medical or surgical treatments such as Pd therapy, intratympanic injection or explorative tympanotomy. The fixed, final hearing was approved if PTA had not been changed for 6 months. PTA was examined at the 9 frequencies $(0.25,0.5,1,1.5,2,3,4,6,8 \mathrm{kHz})$ by a single audiologist, and according to AAO-HNS standard, 1995, an average hearing level was obtained by average of four frequencies: $0.5,1,2,3 \mathrm{kHz}$. If a patient's average hearing level exceeded more than $25 \mathrm{~dB}$, the patient was considered to have hearing loss.

Every affected ears were categorized into either ascending, descending or flat type hearing loss patient according to the formula below.

$$
\alpha=\frac{(500 \mathrm{~Hz}+1 \mathrm{kHz}+2 \mathrm{kHz})}{3}-\frac{(4 \mathrm{kHz}+6 \mathrm{kHz}+8 \mathrm{kHz})}{3}
$$

If $\alpha$ was greater than $15 \mathrm{~dB}$, it was considered as ascending type, if $\alpha$ was lesser than $-15 \mathrm{~dB}$, descending type, and in between them it was categorized as flat type hearing loss. ${ }^{9)}$ Also we classified the patients according to the degree of hearing loss, International Organization for Standardization, 1964; mild (26-40 dB), moderate (41-55 dB), moderately severe (56-70 $\mathrm{dB}$ ), severe (71-90 dB) and profound ( $91 \mathrm{~dB}$ or more). In unilateral hearing loss patients, we also recorded and analyzed the hearing levels of unaffected side and compared them with affected side in each frequency.
Regardless of final hearing level, if a patient obtained more than $15 \mathrm{~dB}$ of hearing gain, the patient was regarded as a improved patient by Siegel's criteria, 1985 (slight recovery or better). ${ }^{10)}$ We investigated the rates of hearing improvement according to each of age, sex, hearing loss type, hearing loss degree and presence of dizziness or tinnitus. To find out if any of those 6 factors are correlated with hearing improvement, we crosstabulated hearing improvement with each of them using $\chi^{2}$ test or Fisher's exact test (SPSS ver. 10.0, SPSS Inc., Chicago, IL, USA). If $p$-value was less than 0.05 , the factor was considered to have statistically significant correlation.

\section{Results}

A total of 40 patients (62 ears) were enrolled. The age ranged from 7 to 69 years with a mean of 49.2. Thirty-two (80\%) patients were male and $8(20 \%)$ were female. Causes of injury were traffic accidents in 35 (87.5\%), assaults in $3(7.5 \%)$ and fall downs in $2(5 \%)$. More specific distributions of age, sex and cause of injury are described in Table 1 . There were 22 (55\%) bilaterally affected and 18 (45\%) unilaterally affected patients. Dizziness and tinnitus were two commonly accompanied symptoms. Dizziness was associated in 20 ears (32\%) whereas tinnitus in 41 ears $(66 \%)$.

The 62 ears were classified into 3 groups according to the shape of PTA. There were 4 (6.5\%) ascending, 34 (54.8\%) descending, 24 (38.7\%) flat type hearing loss. Descending type comprised more than half of all affected ears, which demonstrated that high frequencies were affected more severely than low frequencies. We also calculated and analyzed the average hearing levels of each frequency in every affected ears. It was increased as the frequency reached toward high frequency and this was statistically significant (Repeated Measure ANOVA, $p<0.05$ )(Fig. 1). Even in unaffected side of unilateral hearing loss patients, we could also observe high frequency hearing loss at 3, 4, 6, $8 \mathrm{kHz}$ (Fig. 2). The average hearing levels of unaffected side tended to increase just like those of affected side, and this was also statistically significant $(p<0.05)$. Distribution according to the degree of hearing loss revealed that there were

Table 1. Distribution of patients according to age, sex and cause of injury

\begin{tabular}{|c|c|c|c|c|c|c|c|c|c|c|c|c|c|c|c|}
\hline \multirow{3}{*}{ Injury caused by } & \multicolumn{14}{|c|}{ Age, yr \& sex } & \multirow{3}{*}{ Total } \\
\hline & \multicolumn{2}{|c|}{$0-9$} & \multicolumn{2}{|c|}{$10-19$} & \multicolumn{2}{|c|}{$20-29$} & \multicolumn{2}{|c|}{$30-39$} & \multicolumn{2}{|c|}{$40-49$} & \multicolumn{2}{|c|}{$50-59$} & \multicolumn{2}{|c|}{ Over 60} & \\
\hline & M & $\mathrm{F}$ & M & $\mathrm{F}$ & M & $\mathrm{F}$ & M & $\mathrm{F}$ & M & $\mathrm{F}$ & M & $\mathrm{F}$ & M & $\mathrm{F}$ & \\
\hline Traffic accident & $1(1)$ & 0 & $2(2)$ & 0 & 0 & $1(1)$ & $3(2)$ & 0 & $7(6)$ & $3(1)$ & $5(2)$ & 2 & $9(5)$ & 2 & $35(20)$ \\
\hline Assult & 0 & 0 & 0 & 0 & 1 & 0 & 0 & 0 & 1 & 0 & 1 & 0 & 0 & 0 & 3 \\
\hline Fall down & 0 & 0 & 0 & 0 & 0 & 0 & 0 & 0 & $1(1)$ & 0 & $1(1)$ & 0 & 0 & 0 & $2(2)$ \\
\hline Total & $1(1)$ & 0 & $2(2)$ & 0 & 1 & $1(1)$ & $3(2)$ & 0 & $9(7)$ & $3(1)$ & $7(3)$ & 2 & $9(5)$ & 2 & $40(22)$ \\
\hline
\end{tabular}

Numbers in parentheses indicate the number of bilaterally affected patients. Example: 40 (22) means 22 out of 40 patients were bilaterally affected 
$12(19.4 \%)$ mild, $18(23.0 \%)$ moderate, $11(17.7 \%)$ moderately severe, $10(16.1 \%)$ severe, 11 (17.7\%) profound hearing loss ears. They were relatively evenly distributed and there were no significant correlation between the groups.

There were 15 improved ears in male and 5 in female with a total of $20(32.3 \%)$. Their average age was 45.2 years (15-67 years) and the causes of trauma were all traffic accident. Table 2 shows the rates of hearing improvement according to age, sex, the type of hearing loss, the degree of hearing loss and presence of associated symptoms. According to the type of hearing loss, hearing improvement was observed in 13 (54.2\%) flat type ears, 7 (20\%) descending type ears and no improvement in ascending type hearing loss. Depending on the degree of hearing loss, 6 (33.3\%) moderate, 4 (36.4\%) moderately severe, 4 (40\%) severe, $6(54.5 \%)$ profound hearing loss ears gained hearing improvement, but there was no improvement in 12 mild hearing loss ears. Out of 20 ears associated with dizziness, only 2 (10\%) ears were improved, whereas $16(39 \%)$ out of 41 ears with tinnitus were improved.

Among 20 hearing improved ears, 9 ears showed hearing improvement mainly in low frequencies, one ear in high frequencies, and 10 ears in all frequencies. The amount of hearing gain was varied from 15 to $37.5 \mathrm{~dB}$ with an average of $19.6 \mathrm{~dB}$. In 15 hearing improved ears, the hearing gain was less than 20

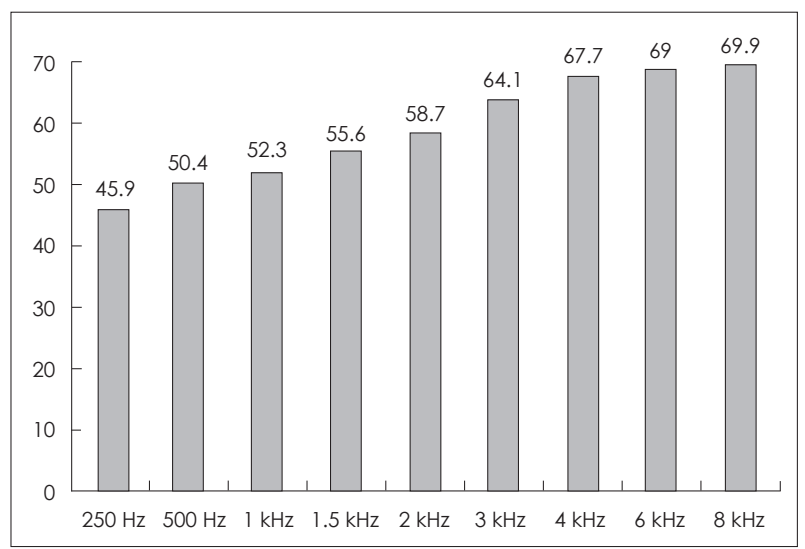

Fig. 1. The average hearing levels of each frequency in all affected ears. The average hearing level was increased as the frequency was reaching toward $8 \mathrm{kHz}$ (Repeated Measure ANOVA, $p<0.05$ ).
dB. Only 5 ears gained greater than $20 \mathrm{~dB}$ and no ear reached within normal range, $20 \mathrm{~dB}$. The detailed profiles of hearing improved ears are shown in Table 3.

Among 6 factors including age, sex, hearing loss type, hearing loss degree and presence of dizziness or tinnitus, only pres-

Table 2. Rates of hearing improvement according to age, sex, the type of hearing loss, the degree of hearing loss and associated symptoms

\begin{tabular}{ccc}
\hline Total & Improved & Rate of \\
number of & number of & improvement \\
ears $(n=62)$ & ears $(n=20)$ & (\%) \\
\hline
\end{tabular}

\section{Age (years)}

$0-9$

$10-19$

$20-29$

$30-39$

$40-49$

$50-59$

Over 60

Sex

Male

Female

2

50.0

33.3

60.0

35.0

33.3

18.8

16

4

3

28.8

Type of hearing loss

Ascending type

Descending type

Flat type

Degree of hearing loss

Mild (26-40 dB)

Moderate (41-55 dB)

Moderately severe

$(56-70 \mathrm{~dB})$

Severe (71-90 dB)

Profound (exceeding

$91 \mathrm{~dB}$ )

Associated symptoms

Dizziness

Present

Absent

Tinnitus

Present

Absent

\section{.}


Table 3. Analysis of patients with hearing improvement

\begin{tabular}{|c|c|c|c|c|}
\hline Patient & $\begin{array}{c}\text { Initial } \\
\text { PTA type }\end{array}$ & $\begin{array}{c}\text { Hearing } \\
\text { improved } \\
\text { frequencies }\end{array}$ & $\begin{array}{c}\text { Initial } \\
\text { degree of } \\
\text { hearing level }\end{array}$ & $\begin{array}{c}\text { Hearing } \\
\text { gain }(\mathrm{dB})\end{array}$ \\
\hline 1 & $\mathrm{~F}$ & Low & $P$ & 27.5 \\
\hline 2 & $\mathrm{~F}$ & All & $P$ & 37.5 \\
\hline 3 & $\mathrm{~F}$ & All & MO & 20.0 \\
\hline 4 & $\mathrm{~F}$ & All & $P$ & 16.3 \\
\hline 5 & $\mathrm{~F}$ & High & S & 15.0 \\
\hline 6 & $\mathrm{~F}$ & Low & $S$ & 15.5 \\
\hline $7 R$ & $\mathrm{~F}$ & Low & MO & 18.7 \\
\hline $7 \mathrm{~L}$ & $\mathrm{~F}$ & All & MO & 17.5 \\
\hline $8 R$ & D & All & MO & 15.0 \\
\hline $8 \mathrm{~L}$ & D & Low & MO & 16.5 \\
\hline $9 R$ & D & Low & MS & 20.0 \\
\hline 9L & D & Low & MS & 17.5 \\
\hline $10 R$ & $\mathrm{~F}$ & All & S & 15.0 \\
\hline $10 \mathrm{~L}$ & D & All & MO & 18.8 \\
\hline $11 R$ & $\mathrm{~F}$ & Low & MS & 16.2 \\
\hline $11 \mathrm{~L}$ & D & All & $S$ & 27.5 \\
\hline $12 R$ & $\mathrm{~F}$ & Low & $P$ & 16.5 \\
\hline $12 \mathrm{~L}$ & D & Low & MS & 16.3 \\
\hline $13 R$ & $\mathrm{~F}$ & All & $\mathrm{P}$ & 22.5 \\
\hline $13 \mathrm{~L}$ & $\mathrm{~F}$ & All & $P$ & 22.5 \\
\hline
\end{tabular}

R: right ear, L: left ear, F: flat, D: descending, MO: moderate, MS: moderately severe, S: severe, P: profound, PTA: pure tone audiometry

ence of dizziness had correlation with hearing improvement. The patients without vertigo had more chance to recover their hearing, and this was statistically significant $(p=0.01)$.

\section{Discussion}

Various studies have tried to explain the hearing loss that appears after head trauma, but the mechanism of injury is not clear yet. Brunner ${ }^{5)}$ reported his experiments with guinea pigs. Intentional head blows were made to the guinea pigs, and they all demonstrated inner ear hemorrhage which is believed to be responsible for the hearing loss. On the other hand, Wittmaack assumed that a travelling pressure wave injured inner ear structures directly. He produced shock pulses in the fluids of the labyrinth in cats, and found loss of hair cells and spiral ganglion cells in the middle turns of cochlea. He also found a few fractures in the spiral lamina, damage to the basal membrane, and damage in the perilymphatic spaces. Based on those experiments, it is assumed that compression and vibration forces generated by head trauma are transmitted through skull to inner ear, and produce a shock pulse in the inner ear. This pulsation of the inner ear fluids may displace the basilar membrane and cause injury to the organ of Corti, creating damage to the inner ear hair cell, laceration of the membranous labyrinth and hemorrhages in various locations. ${ }^{6-8)}$

The purpose of this study was to determine the characteristics of audiometry and prognostic factors in the labyrinthine concussion patients. The results of this study showed that most affected ears were presented as descending or flat type hearing loss, and the hearing deficit was more severe in high frequencies. This result corresponds well with that of the early study which reported by Schuknecht, et al. ${ }^{11)}$ who advocated that sensorineural hearing loss was maximal in the 4000 to $6000 \mathrm{~Hz}$ in head blowing injuries. He recorded the hearing losses and pathological changes in cats subjected to head blows. The postconcussion behavioral audiogram revealed hearing losses most severely in high tones, and in histological studies, damage of organ of Corti was most severe in the middle part of the basal turn which serves 4000 to $8000 \mathrm{~Hz}$.

Ulug and Ulubil ${ }^{4)}$ reported three patients with contra-lateral labyrinthine concussion in unilateral temporal bone fracture, and conjectured that high pressure waves caused by a severe blow to the head are directly transmitted to the cochlea by bone conduction, very similar to acoustic trauma. Ulug's case report shows the similarity to result of this study, high frequency hearing loss of unaffected ear in unilateral hearing loss patients. This indicates that the vibration forces generated by head trauma is transmitted through bone conduction even to the other side of the head and bring sensorineural hearing loss.

In the analysis of hearing improved patients, hearing gain was obtained mostly in low frequencies rather than high frequencies. We assumed that it was because the vibrating wave influenced the basal turn of cochlea most severely and resulted irreversible damage to the organ of Corti in basal turn.

Dizziness was accompanied with hearing loss in $32 \%$ of enrolled subjects. Dizziness or vertigo derived by blunt head trauma can be explained by following several mechanisms ${ }^{1)}$ and some of these mechanisms could contribute to hearing loss. First, shearing effect on the root of eighth nerve, where the nerve enters to the brain stem, brings petechial hemorrhages and leads to uncompensated vestibular disturbance and sensorineural hearing loss. Second, just like damage to cochlear, direct injury to vestibular end organs, semicircular canals and vestibule, may take a part in dizziness developing. It is assumed that travelling waves from intracranial cerebrospinal fluid following head trauma disrupts the sensory epithelium of vestibular end organs. Third, benign paroxysmal positional vertigo after head trauma has been reported frequently. ${ }^{12,13)}$ Forth, dizziness can be developed by endolymphatic duct disruption which eventually leads to endolymphatic hydrops, Meniere's syndrome. Not only direct disruption of endolymphatic duct but also obstruction of endolymphatic duct or sac with blood clot or otoconial debris causes the same consequences. ${ }^{1)}$ Fifth, traumatic perilymphatic 
fistula can be developed either in the fistula ante fenestra of the oval window or in the round window. This study proved that the ears without dizziness have more chance to recover hearing, even if the amount of hearing gain is not fully sufficient. However not all of the above five mechanisms are correlated with hearing loss in this study. In the process of patient selection, we made clear that patients with brain stem hemorrhage and perilymphatic fistula were excluded. Then the result of this study can be explained by following. The stronger transmitting force generates more damage to hair cells of both cochlear and vestibular organs, and makes more chance to build up otoconial debris or blood clot. Thus the stronger force causes the more chance to develop irreversible sensorineural hearing loss, and at the same time dizziness due to Meniere's syndrome, paroxysmal positional vertigo or direct vestibular organ damage. However, it has not been mentioned previously at the results, we could not find any correlation between the presence of dizziness and the degree of initial hearing loss. Another limitation is that this study could not completely distinguish post- concussion hearing loss from presbycusis.

\section{Conclusion}

In labyrinthine concussion patients, the hearing loss is severer in high frequencies rather than low frequencies, and even in unaffected ears, high frequencies hearing losses are observed. The hearing gain is obtained mainly in low frequencies, and there is greater possibility of hearing gain in ears without diz- ziness.

\section{REFERENCES}

1) Fitzgerald DC. Head trauma: hearing loss and dizziness. J Trauma 1996;40:488-96.

2) Grove WE. Skull fractures involving the ear. A clinical study of 211 cases. Laryngoscope 1939;49:678-707, 833-70.

3) Canalis RF, Lambert PR. The ear: comprehensive otology. Philadelphia (PA): Lippincott Williams \& Wilkins;1999. p.795-6.

4) Ulug T, Ulubil SA. Contralateral labyrinthine concussion in temporal bone fractures. J Otolaryngol 2006;35:380-3.

5) Brunner H. Disturbances of the function of the ear after concussion of brain. Laryngoscope 1940;50:921-49.

6) Schuknecht HF, Davison RC. Deafness and vertigo from head injury. AMA Arch Otolaryngol 1956;63:513-28.

7) Kochhar LK, Deka RC, Kacker SK, Raman EV. Hearing loss after head injury. Ear Nose Throat J 1990;69:537-42.

8) Bergemalm PO. Progressive hearing loss after closed head injury: a predictable outcome? Acta Otolaryngol 2003;123:836-45.

9) Ventry IM, Chaiklin JB. Evaluation of pure tone audiogram configurations used in identifying adults with functional hearing loss. J Aud Res 1965;5:212-8.

10) Siegel LG. The treatment of idiopathic sudden sensorineural hearing loss. Otolaryngol Clin North Am 1975;8:467-73.

11) Schuknecht HF, Neff WD, Perlman HB. An experimental study of auditory damage following blows to the head. Ann Otol Rhinol Laryngol 1951;60:273-89.

12) Bertholon P, Chelikh L, Tringali S, Timoshenko A, Martin C. Combined horizontal and posterior canal benign paroxysmal positional vertigo in three patients with head trauma. Ann Otol Rhinol Laryngol 2005;114:105-10.

13) Motin M, Keren O, Groswasser Z, Gordon CR. Benign paroxysmal positional vertigo as the cause of dizziness in patients after severe traumatic brain injury: diagnosis and treatment. Brain Inj 2005;19: 693-7. 